\title{
Microanalysis of variable-interval performance during stimulus compounding
}

\author{
M. CATHERINE BUSHNELL and STANLEY J. WEISS \\ The American University, Washington, D.C. 20016
}

\begin{abstract}
Additive summation is observed when more responses are emitted to the simultaneous presentation (tone-plus-light) of independently conditioned stimuli (tone and light) than to either stimulus presented alone. The current experiment sought to determine if this increased rate during tone-plus-light was a function of a new modal interresponse time (IRT) or a differential mixing of pauses with a modal IRT characteristic of the responding in tone and light alone. Three rats were trained on a three-component multiple schedule where tone and light were each associated with a variable-interval 30 -sec schedule while a variable-interval 100 -sec operated in the simultaneous absence of these stimuli, tone-off and light-out. Baseline response rates were 2-4 times as high in tone or light as in their absence. In testing, more responses were emitted to tone-plus-light than to tone or light by all animals, but the modal IRT was in the 0.2-0.4-sec IRT bin for all test conditions. Tone-plus-light controlled fewer long IRT values and more responses in the short modal category than tone or light alone. These results support the response mixing hypothesis of stimulus control; i.e., no "new" behavior was observed during the novel combination of stimulus elements, only a mixture of previously reinforced behavior patterns in different proportions.
\end{abstract}

On a generalization test, stimulus control of behavior can be assayed in molar terms, i.e., as average response rates, and/or in molecular terms, i.e., as interresponse times (IRTs). In molar terms, a systematic relationship exists between response rates to the generalized stimuli and the similarity of these stimuli to the training stimulus $\left(\mathrm{S}^{\mathrm{D}}\right)$, with the peak at the $S^{D}$ value (Guttman \& Kalish, 1956). Are these lowered response rates in the presence of the generalized stimuli merely a mixing of previously reinforced behaviors with pauses or are the behavior patterns unique to those stimuli? In order to answer this question, Blough (1963), Crites, Harris, Rosenquist, and Thomas (1967), and Sewell and Kendall (1965) collected IRT data during stimulus generalization tests. Each found that the stimulus generalization gradient was produced by an increase in the number of long IRTs, while the modal IRT value remained unchanged. They concluded that the rates to the generalized stimuli were a product of mixing the short IRT's characteristic of $S^{D}$ responding with long pauses.

Other investigators demonstrated the mixing of behavior more explicitly. Cumming and Eckerman (1965), Migler (1964), Migler and Millenson (1969),

\footnotetext{
This experiment was supported by Grant MH-16853 to the second author from the National Institute of Mental Health, United States Public Health Service. It was presented at the 1976 Eastern Psychological Association Convention in New York. We would like to thank Dr. Alan M. Silberberg for his critical comments and advice on earlier versions of this manuscript. Reprints may be obtained from Stanley J. Weiss, The American University, Department of Psychology, Washington, D.C. 20016.
}

and Wildemann and Holland (1972) each trained animals to produce discrete response patterns in the presence of two stimulus values. Subsequent generalization tests included stimuli intermediate in value to the two training stimuli. Bimodal distributions of IRTs were obtained, with each mode representing one of the discrete response patterns emitted in the presence of the two training stimuli. Varying response rates in the presence of the intermediate test stimuli were generated by differential proportions of responses distributed to each response mode.

If attenuated response rates in the presence of stimuli intermediate to the training stimuli are a product of mixing previously learned behavior patterns, could this "mixing" also account for the attenuated responding (response averaging) observed when two training stimuli are presented simultaneously? The stimulus compounding assay provides a means of determining this. When a high response rate is conditioned to one stimulus and a low response rate is conditioned to a second stimulus, an intermediate response rate is obtained when the two stimuli are presented concurrently (Adams \& Allen, 1971; Weiss, 1967). Weiss (1972a) determined that, during compound stimulus presentations, the obtained intermediate response rate was actually a mixing of high-rate responding associated with one training stimulus and low-rate responding associated with the other training stimulus. No "intermediate" behavior was revealed.

Intermediate response rates are not always the resultant behavior when two discriminative stimuli 
are presented in compound. If each of two stimuli (tone and light) control a moderate response rate while the absence of both stimuli, tone off and light out, controls a lower rate, more responses are emitted during tone-plus-light $(T+L)$ test presentations than during either tone or light alone (additive summation) (Weiss, 1964, 1971, Experiment 2; Wolf, 1963). Is the increased response rate during $\mathrm{T}+\mathrm{L}$ a function of a new modal IRT value or a differential mixing of pauses with a modal IRT characteristic of tone or light responding? No IRT data have been collected when a novel stimulus configuration controls an enhanced response rate, as when additive summation is observed. The present experiment sought to determine if an enhanced response rate to the novel $\mathrm{T}+\mathrm{L}$ stimulus condition was the product of a recombination of IRT patterns observed in training during tone or light alone. If this were the case, while the modal IRT value maintained its integrity over tone, light, and $\mathrm{T}+\mathrm{L}$, $\mathrm{T}+\mathrm{L}$ would control fewer long pauses than tone or light. To evaluate this hypothesis, IRT data were collected under conditions where additive summation was obtained during compound stimulus presentations.

\section{METHOD}

\section{Subjects}

Three adult male Long-Evans (hooded) rats were trained and tested at $80 \%$ of their approximately $350 \mathrm{~g}$ free-feeding weights. All subjects were individually housed and had continual access to water in their home cages.

\begin{abstract}
Apparatus
A single-lever operant-conditioning chamber, housed in a sound-attenuating chest, was employed. Two discriminative stimuli, a $2,000-\mathrm{Hz}$ tone and a $130.2-\mathrm{cd} / \mathrm{m}^{2}$ light, were used. The speaker for the tone was located $23 \mathrm{~cm}$ above the chamber, and the light was provided by two $120-\mathrm{V}, 25-\mathrm{W}$ bulbs located $9 \mathrm{~cm}$ on either side of the chamber. The intensity of the tone at the grid floor was 90-95 dB, measured by a General Radio Type 1565-A sound-level meter using the $C$ scale. An exhaust fan provided ambient noise of approximately $80 \mathrm{~dB}$, while a shiclded $7.5 \mathrm{~W}$, 120-V houselight kept the chamber at least dimly illuminated at all times. Similar chambers, chests, and stimuli are described in detail by Weiss $(1969,1970)$. A PDP-8e digital computer, located in a room adjacent to that containing the experimental chamber, was used to program the scheduled contingencies and to collect response data. Reinforcers were Noyes $45-\mathrm{mg}$ rat pellets.
\end{abstract}

\section{Procedure}

Baseline training. On Day 1, rats were trained to approach the food trough at the sound of the feeder click. On Day 2, the barpress response was shaped, and the subjects were then permitted to earn approximately 150 pellets on a continuous reinforcement schedule. In subsequent sessions, the subjects received food on a variable-interval (VI) schedule in which the first leverpress occurring a mean time, defining the VI, after the last reinforced response produced a food pellet. On Day 3, a VI 15-sec schedule was employed, and on subsequent sessions the mean VI value was increased to $30,45,60,75,90$, and finally $100 \mathrm{sec}$. By Session 15, each rat was responding on a VI 100-sec schedule. During this phase of training, only the very dim houselight was on (tone off and light out, $\overline{\mathrm{T}}+\overline{\mathrm{L}}$, condition). When the subject was responding regularly, after approximately $10 \mathrm{VI} 100$-sec sessions, a threecomponent multiple schedule was introduced. On this schedule, leverpressing was reinforced according to a VI $30-\mathrm{sec}$ schedule during tone and during light, while leverpressing continued to be reinforced according to a VI 100 -sec schedule in $\bar{T}+\overline{\mathrm{L}} \cdot \overline{\mathrm{T}}+\overline{\mathrm{L}}$ was present during $50 \%$ of the total session time and always followed tone or light components. Either tone or light, randomly, could follow a $\bar{T}+\bar{L}$ period. Component durations unsystematically varied from 1 to $10 \mathrm{~min}$ with an average duration of $4 \mathrm{~min}$. The subjects received at least 902 -h sessions of training before testing.

Extinction stimulus-compounding test. This test consisted of 12 block-randomized presentations of tone, light, and tone-pluslight $(T+L)$. Two minutes of $\bar{T}+\bar{L}$ followed each 2 -min presentation of tone, light, or $\mathrm{T}+\mathrm{L}$. Reinforcement was discontinued during this 144-min test. Before the test began, the subject received a warm-up of approximately $1 / 2 \mathrm{~h}$ of regular baseline training. IRT data were collected in 0.2 -sec bins during the test and during selected terminal training sessions.

\section{RESULTS}

\section{Response-rate Analysis}

During baseline training, all subjects emitted responses at approximately equal rates in tone and in light. These tone/light rates were $31.5 / 34.6$, $49.6 / 46.3$, and $54.6 / 57.5$ responses/min over the final four sessions for subjects 217,218 , and 230 , respectively. These average rates in tone or light were always more than twice that in $\bar{T}+\bar{L}$. The $\bar{T}+\bar{L}$ response rates were $43.8 \%, 27.8 \%$, and $37.8 \%$ of the average light or tone response rates for subjects 217 , 218 , and 230 , respectively.

Figure 1 shows that in the extinction test all subjects emitted more responses during $T+L$ than during tone or light (additive summation). The response rates to $T+L$ relative to the mean of the rates to tone and to light were $1.4,2.3$, and 2.1 for subjects 217,218 , and 230 , respectively.

\section{Interresponse Time Analysis}

The IRT distributions for each extinction test stimulus are presented in Figures 2 and 3. For clarity, the 0.2 -sec bins are collapsed into 1 -sec bins. Figure 2 shows short IRTs (0-1) and long pauses (greater than $9 \mathrm{sec}$ ). At least $75 \%$ of each subject's responses fell in these two categories. For every subject, the largest

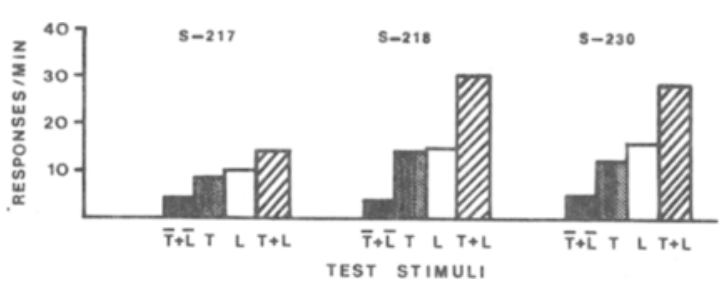

Figure 1. Response rates (responses per minute) to $\mathbf{T}+\mathbf{L}$, tone, light, and $T+L$ during the extinction stimulus-compounding test. 
A
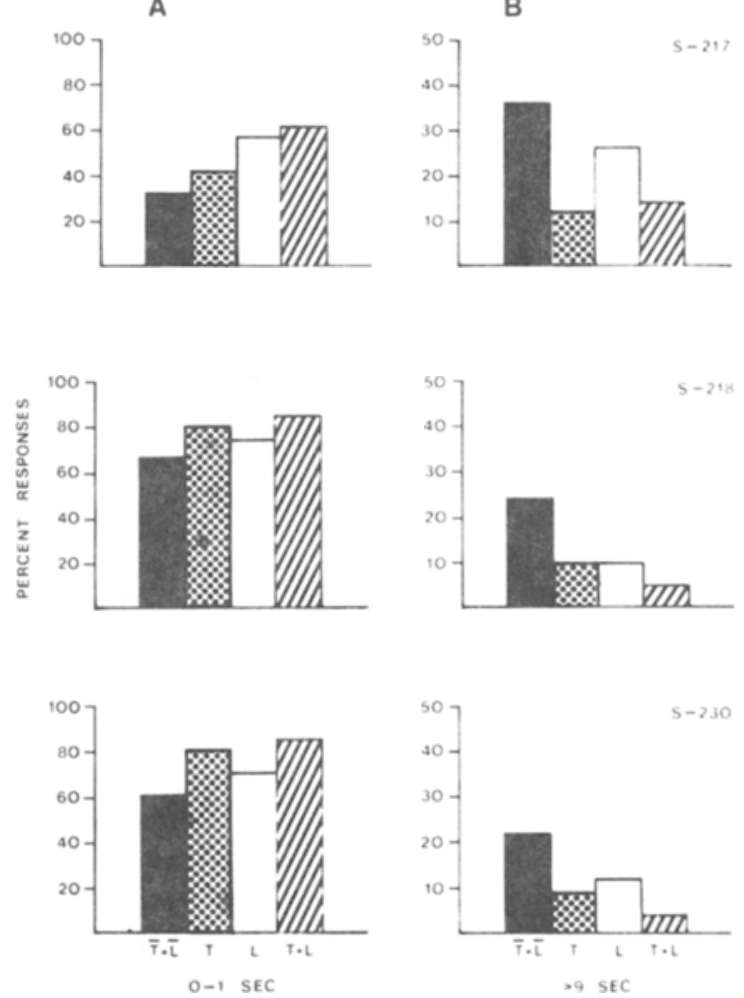

Figure 2. Within each stimulus condition, the percentage of responses emitted within $1 \mathrm{sec}$ of each other (A) and with IRTs of more than $9 \mathrm{sec}(B)$ during the extinction test. Note that the scale of the 0-1-sec ordinate is twice that of the $>9-$ sec ordinate.

percentage of responses distributed to the $0-1-\mathrm{sec}$ category was obtained during $T+L$ and the smallest percentage during $\overline{\mathrm{T}}+\overline{\mathrm{L}}$. The probability that, for all these subjects, $T+L$ ranks first and $\bar{T}+\bar{L}$ ranks fourth, under the null hypothesis that all rankings of the four conditions are equally likely, is .001 . Following a long pause, $\overline{\mathrm{T}}+\overline{\mathrm{L}}$ controlled the greatest percentage of responses for each subject. In all but one case, the smallest percentage was distributed to this category during $\mathrm{T}+\mathrm{L}$. Under the null hypothesis that all rankings are equally likely, the probability that for two of three subjects $\bar{T}+\overline{\mathrm{L}}$ ranks first and $T+L$ ranks fourth is .02 . Rat 217 emitted a slightly larger percentage of responses in the greater-than-9-sec category during $\overline{\mathrm{T}}+\overline{\mathrm{L}}$ $(13.8 \%)$ than during tone $(11.6 \%)$. This subject also showed the weakest additive summation, with the response rate in light being closest to that in $\mathrm{T}+\mathrm{L}$ (see Figure 1). Figure 3 shows that the percentage of responses in the intermediate IRT categories is indistinguishable among stimulus conditions for each subject, with less than $5 \%$ customarily contained in any category.

Examinations of the IRT distributions in Figures 2A and $2 \mathrm{~B}$ reveals that the modal IRT was in the $0-1-\mathrm{sec}$ category for each extinction test stimulus condition. This means that the modal IRT for the different conditions could be in any one of the five $0.2-\mathrm{sec}$ bins collapsed to produce the category. Therefore, to determine whether small differences exist in modal IRT values among conditions, these IRT data are presented in 0.2 -sec bins in Table 1. Only the first three bins $(0-0.2,0.2-0.4$, and $0.4-0.6 \mathrm{sec})$ are presented, as the data for the longer IRT values look almost identical whether grouped into $0.2 \mathrm{sec}$ or 1.0 -sec bins. Table 1 reveals that the modal IRT value falls in the $0.2-0.4-\mathrm{sec}$ bin for every subject in each stimulus condition, including $T+L$. For each rat, the proportion of responses emitted in the $0.2-0.4-\mathrm{sec}$ bin is smallest in $\bar{T}+\overline{\mathrm{L}}$ and largest in $T+L$.

IRT data collected during training were similar to those collected during the extinction test. Table 2 shows a modal IRT of 0.2-0.4 sec for each subject in every stimulus condition on the final training days before testing. As in the extinction test, more long pauses and fewer short modal IRTs were obtained during $\bar{T}+\bar{L}$ than during tone or light. The distributions for tone, light, and $\overline{\mathrm{T}}+\overline{\mathrm{L}}$ within the 1-9-sec IRT categories were indistinguishable from those in Figure 3.

Table 1

Percentage of Extinction Test Responses With IRTs Between 0 and $.6 \mathrm{Sec}$

\begin{tabular}{ccccc}
\hline \multirow{2}{*}{ Subject } & & \multicolumn{3}{c}{ IRT Value } \\
\cline { 3 - 5 } & Stimulus & $0-.2$ & $.2-.4$ & $.4-.6$ \\
\hline \multirow{3}{*}{217} & $\overline{\mathrm{T}}+\overline{\mathrm{L}}$ & 1.5 & 17.0 & 8.9 \\
& Tone & 3.1 & 23.7 & 8.3 \\
& Light & 2.5 & 36.1 & 10.8 \\
& $\mathrm{~T}+\mathrm{L}$ & 3.2 & 36.3 & 14.5 \\
\multirow{3}{*}{218} & $\overline{\mathrm{T}}+\overline{\mathrm{L}}$ & 6.1 & 32.3 & 19.2 \\
& Tone & 5.7 & 43.0 & 24.2 \\
& Light & 7.0 & 38.6 & 18.4 \\
& T $+\mathrm{L}$ & 5.5 & 47.7 & 20.8 \\
& $\bar{T}+\overline{\mathrm{L}}$ & 10.2 & 27.5 & 16.1 \\
& Tone & 12.7 & 40.4 & 21.8 \\
& Light & 8.8 & 33.9 & 20.0 \\
& T $+\mathrm{L}$ & 12.2 & 41.1 & 19.4 \\
\hline
\end{tabular}

Table 2

Percentage of Short IRTs and Long Pauses in the Final Training Session

\begin{tabular}{cccccccc}
\hline & & \multicolumn{7}{c}{ IRT Value } \\
\cline { 3 - 8 } Subject & Stimulus & $\mathbf{0 - . 2}$ & $.2-.4$ & $.4-.6$ & $.6-.8$ & $.8-1.0$ & $>9.0$ \\
\hline \multirow{3}{*}{217} & $\overline{\mathrm{T}}+\overline{\mathrm{L}}$ & 1.8 & 43.8 & 12.3 & 2.9 & 1.6 & 7.6 \\
& Tone & 3.2 & 49.9 & 11.3 & 2.9 & 1.1 & 3.2 \\
& Light & 5.3 & 51.5 & 11.1 & 1.0 & .5 & 2.2 \\
\multirow{4}{*}{218} & $\overline{\mathrm{T}}+\overline{\mathrm{L}}$ & 3.2 & 34.3 & 24.6 & 9.7 & 4.2 & 10.5 \\
& Tone & 4.6 & 35.7 & 27.3 & 9.6 & 5.1 & .8 \\
& Light & 5.7 & 37.8 & 24.0 & 9.7 & 4.6 & 1.6 \\
\multirow{2}{*}{230} & $\overline{\mathrm{T}}+\overline{\mathrm{L}}$ & 3.0 & 21.6 & 12.3 & 3.7 & 1.4 & 2.6 \\
& Tone & 7.3 & 50.9 & 24.2 & 4.8 & 1.3 & .8 \\
& Light & 4.6 & 43.2 & 28.3 & 7.9 & 2.0 & 1.7 \\
\hline
\end{tabular}



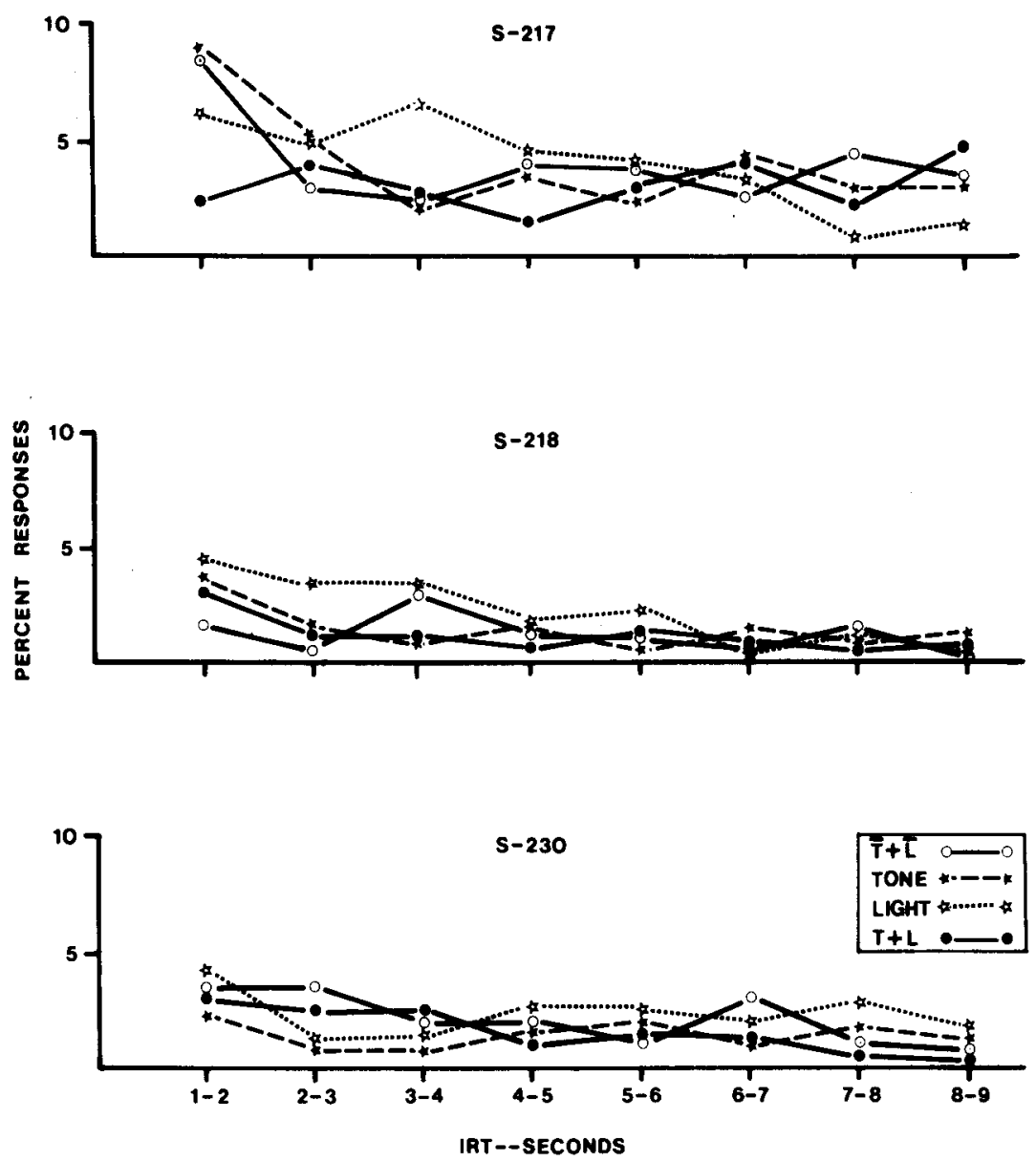

Figure 3. The percentage of total test responses falling in the intermediate IRT categories, i.e., $1<$ IRT $<9$ sec. These are shown in 1-sec bins.

\section{IRT Per Opportunity Analysis}

An IRT per opportunity transformation (Anger, 1956) was performed on the test data to determine the conditional probability of a response, given that a specified time has elapsed since the preceding response. Graphically, a function horizontal to the $\mathrm{X}$-axis indicates that response probability is unaffected by time since the preceding response, suggesting that successive responses are independent. Figure 4 shows that for every stimulus condition, each subject showed a mode at short IRT values. Other than these modes, the curves are relatively flat, indicating response independence for longer IRT values.

\section{DISCUSSION}

The heightened response rate in $\mathrm{T}+\mathrm{L}$ (additive summation) appears to be due to a decrease in long IRT values and an increase in the proportion of responses distributed at the short modal value, relative to the distributions to tone and light alone. These results support the response-mixing hypothesis of stimulus control; i.e., no "new" behavior was ob- served during the novel combination of stimulus elements, only a mixture of previously reinforced behavior patterns in different proportions. While previous research supports the response-mixing hypothesis when test stimuli engender response rates lower than or intermediate to those controlled by the training stimuli, this is the first report of response mixing in the presence of stimuli which control higher response rates than those controlled by the training stimuli. Consequently, a microanalysis of responding (IRTs) reveals that organisms are mixing previously learned behavior patterns even when a macroanalysis (i.e., response rates) reveals no apparent response mixing as would be indicated by a response averaging. The increased rates to combined stimuli observed during stimulus compounding and the decreased rates to generalized stimuli observed during stimulus generalization both appear to be the product of differential mixing of the short modal IRTs and long pauses. $^{1}$

\section{Comparison of VI-Produced IRTs}

The present study provides a within-subject comparison of IRTs generated by different VI 

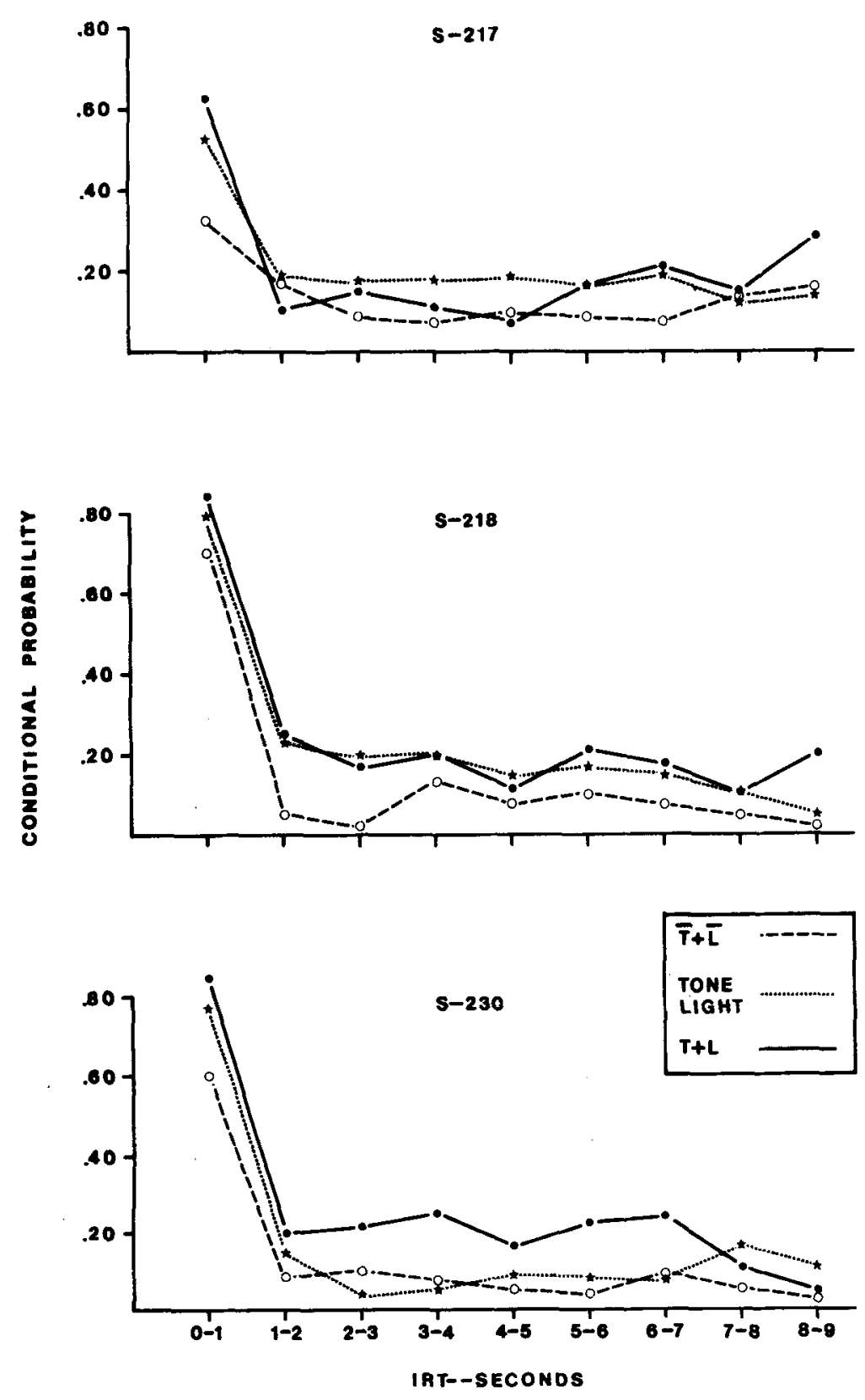

Figure 4. Distributions of conditional probability of a response, given that a certain time has elapsed since the previous response. Individual distributions for each subject are shown for $T+L$, the average of tone and light, and $T+L$ for the test data in Figures 2 and 3.

schedules of food presentation. Several investigators (Anger, 1956; Crites, et al., 1967; Kintsch, 1965) have found that rats produce a short modal IRT value when leverpressing for food on VI schedules. Blough and Blough (1968) analyzed IRT data of pigeons responding on VI schedules and found two-state performance similar to that obtained in the present investigation. Response "bursts" were separated by pauses by various lengths. The present study extends these findings by showing that the modal IRT is invariant over VI values within a multiple schedule of VI reinforcement. When re- sponding on either a VI 30 -sec or VI 100-sec schedule, rats tend to leverpress at a unitary, rapid rate. These response bursts are separated by pauses by varying duration. The different response rates produced during the VI 30-sec and VI 100-sec schedules appear to be a function of the number of these bursts and the duration of the pauses.

\section{Theoretical Implications}

The present finding-what appears to be "new" behavior in the presence of $T+L$ is actually a mixing of previously learned behavior-is consistent 
with a composite-stimulus control model proposed by Weiss (1969, 1972b). According to Weiss' model, the absence, as well as the presence, of a stimulus can control behavior. The use of tone and light allows four binary combinations of discriminative stimuli: tone and light-out $(T+\overline{\mathrm{L}})$, light and toneoff $(\mathrm{L}+\overline{\mathrm{T}})$, tone-off and light-out $(\overline{\mathrm{T}}+\overline{\mathrm{L}})$, and tone-plus-light $(T+L)$. If $\bar{T}+\bar{L}$ is associated with a low rate of responding (VI 100-sec schedule), then during tone $(T+\bar{L})$, one stimulus modality $(T)$ controls a high response rate appropriate to a VI 30-sec schedule, while the other stimulus modality (L) controls a low response rate appropriate to the $\overline{\mathrm{T}}+\overline{\mathrm{L}}$ stimulus condition. The resultant behavior during $T+\overline{\mathrm{L}}$ is then a "mix" of high-rate responding controlled by $T$ and low-rate responding controlled by $\overline{\mathrm{L}}$. Similarly, during light $(\mathrm{L}+\overline{\mathrm{T}})$, the final behavior is a mixture of high-rate responding under the control of $\mathrm{L}$ and low-rate responding under the control of $\overline{\mathrm{T}}$. Consequently, a substantial number of long pauses, characteristic of $\bar{T}+L$ responding, are obtained during tone and light presented separately. However, when $T+L$ is presented, only stimulus conditions that control high rates are present and the number of long pauses, controlled by $\overline{\mathrm{T}}$ or $\overline{\mathrm{L}}$, decreases. While the short modal IRT value (controlled by $\mathrm{T}$ or $\mathrm{L}$ ) remains unchanged, the proportion of responses in this category increases, leading to additive summation. The present results support this composite-stimulus control model.

\section{REFERENCES}

Adams, D. L., \& Allen, J. D. Compound stimulus control by discriminative stimuli associated with high and moderate response rates. Journal of the Experimental Analysis of Behavior. 1971, 16. 201.205.

ANGER. D. The dependence of interresponse times upon the relative reinforcement of different interresponse times. Journal of Experimental Psychology, 1956. 52, 145-161.

BLough. D. S. Interresponse time as a function of continuous variables: A new method and some data. Journal of the Experimental Analysis of Behavior. 1963. 6, 237-246.

Blovgh, P. M.. \& Blough. D. S. The distribution of interresponse times in the pigeon during variable-interval reinforcement. Journal of the Experimental Analysis of Behavior, 1968. 11, 23-27.

Crites. R. J., Harris, R. T., Rosenquist, H., \& Thomas, D. R. Response patterning during stimulus generalization in the rat. Journal of the Experimental Analysis of Behavior, 1967, 10. 165-168.
Cumminc, W. W., \& Eckerman, D. A. Stimulus control of a differential operant. Psychonomic Science, 1965, 3, 313-314.

Guttman, N., \& Kal.ish, H. I. Discriminability and stimulus generalization. Joumal of Experimental Psychology, 1956, 51. 79-88.

KINTSCH. W. Frequency distribution of interresponse times during VI and VR reinforcement. Joumal of the Experimental Analysis of Behrvior, 1965, 8, 347-352.

Migler, B. Effects of averaging data during stimulus generalization. Joumal of the Experimental Analysis of Behavior, 1964, 7. 305-307.

Migler. B., \& Millenson, J. R. Analysis of response rates during stimulus generalization. Journal of the Experimental Analysis of Behavior, 1969, 12, 81-87.

Sewell, W. R., \& Kendall, S. B. A note on interresponse time distributions during generalization testing. Psychonomic Science, 1965, 3, 95-96.

WEISS, S. J. Summation of response strengths instrumentally conditioned to stimuli in different sensory modalities. Journal of Experimental Psychology, 1964, 68, 151-155.

WEISs, S. J. Free-operant compounding of variable-interval and low-rate discriminative stimuli. Journal of the Experimental Analysis of Behavior. 1967, 10, 535-540.

WEISS, S. J. Attentional processes along a composite stimulus continuum during free-operant summation. Joumal of Experimental Psychology, 1969, 82, 22-27.

WEISS, S. J. An effective and economical sound-attenuation chamber. Joumal of the Experimental Analysis of Behavior. 1970, 13, 37-39.

WE1ss. S. J. Discrimination training and stimulus compounding: Consideration of non-reinforcement and response differentiation consequences of $S \Delta$. Journal of the Experimental Analysis of Behavior, 1971, 15, 387-402.

WEIss, S. J. Free-operant compounding of high- and low-rate discriminative stimuli: An interresponse time analysis. Learning and Motivation. 1972, 3. 469-478. (a)

Weiss, S. J. Stimulus compounding in free-operant and classical conditioning: A review and analysis. Psychological Bulletin, 1972, 78. 189-208. (b)

Wildemann, D. G., \& Holland, J. G. Control of a continuous response dimension by a continuous stimulus dimension. Journal of the Experimental Analysis of Behavior, 1972, 18, 419-434.

Wolf. M. M. Some effects of combined SD's. Joumal of the Experimental A nalysis of Behavior, 1963, 6, 343-347.

\section{NOTE}

1. In the present experiment, restrictions were not placed on reinforced IRTs, and the same short modal IRT was obtained to the $S^{D}$ associated with the different VI values and to these $S^{D_{S}}$ combined. If a somewhat longer modal IRT were generated by delivering reinforcement only following a specific IRT value, during stimulus compounding subjects' modal IRTs might be shifted to shorter values characteristic of nonpaced schedules. This remains to be determined.

(Received for publication March 22, 1977; revision accepted July 20,1977 .) 